\title{
Association of hypercholesterolemia and cardiac function evaluated by speckle tracking echocardiography in a rabbit model
}

\author{
Liyun Liu, Yuming Mu*, Wei Han and Chunmei Wang
}

\begin{abstract}
Background: Although hypercholesterolemia is a major risk factor for coronary artery disease (CAD), only limited data are available regarding its direct effect on myocardial function apart from CAD. The aim of this study was to evaluate LV systolic function using speckle-tracking echocardiography and investigate the relationship between hypercholesterolemia and myocardial function.
\end{abstract}

Methods: Twenty-eight rabbits were randomly divided into three groups: 8 were fed normal chow for 3 months (group 1) and the remaining 20 were fed an atherogenic diet for 2 (group 2) or 3 months (group 3). Global systolic radial, circumferential and longitudinal peak strain were calculated. Serum total cholesterol (TC), low density lipoprotein cholesterol (LDL-C) and myocardial cholesterol levels were measured.

Results: Global systolic longitudinal strain were both decreased in the group 2 and $3(P<0.001)$, whereas radial strain were increased $(P<0.001)$ compared with group 1. Global circumferential strain in the group 3 was significantly reduced $(P<0.001)$. Serum and myocardial cholesterol concentration markedly increased in the group 2 and group $3(P<0.001)$. There was a significant inverse correlation between longitudinal strain and serum TC, LDL-C as well as myocardial cholesterol levels $(r=-0.723, r=-0.794, r=-0.700, P$ both $<0.001)$. A significant negative correlation was also noted between circumferential strain and serum TC, LDL-C as well as myocardial cholesterol levels $(r=-0.518, P=0.007 ; r=-0.691, P<0.001 ; r=-0.659, P<0.001)$. A significant positive correlation was found between radial strain and serum TC, LDL-C as well as myocardial cholesterol levels $(r=0.432, P=0.028$; $r=0.602, P=0.001 ; r=0.469, P=0.016)$.

Conclusion: Although LV morphology and ejection fractions were not different among the three groups, elevated concentration of cholesterol, especially in serum LDL-C, was significantly associated with LV systolic dysfunction. The findings also indicate that reductions in longitudinal was the first appeared, followed by circumferential, and was compensated for by increasing radial strain.

Keywords: Hypercholesterolemia, Myocardial function, Speckle tracking echocardiography

\section{Background}

Although hypercholesterolemia has emerged as a strong risk factor for coronary artery disease (CAD) [1-3], only limited data are available regarding its direct effect on myocardial function apart from CAD [4-6]. The metabolic derangement of hypercholesterolemia can result in abnormalities of cardiac function that are

\footnotetext{
*Correspondence: mym1234@126.com

Department of Echocardiography, Center of Medical Ultrasound, First Affiliated Hospital of Xinjiang Medical University, No. 137, Li Yu Shan South Road, Urumai 830011, China
}

likely independent of effects on the vasculature [5]. While single left ventricular (LV) myocytes isolated from hypercholesterolemic rabbits demonstrated a significant reduction in systolic function without any change in blood pressure or LV morphology [4], few data are available from in vivo investigations.

Speckle tracking echocardiography (STE), a relatively new echocardiographic imaging modalities, offers an objective and quantitative evaluation of global and regional myocardial deformation in longitudinal, radial and circumferential directions [7-9]. A large amount of 


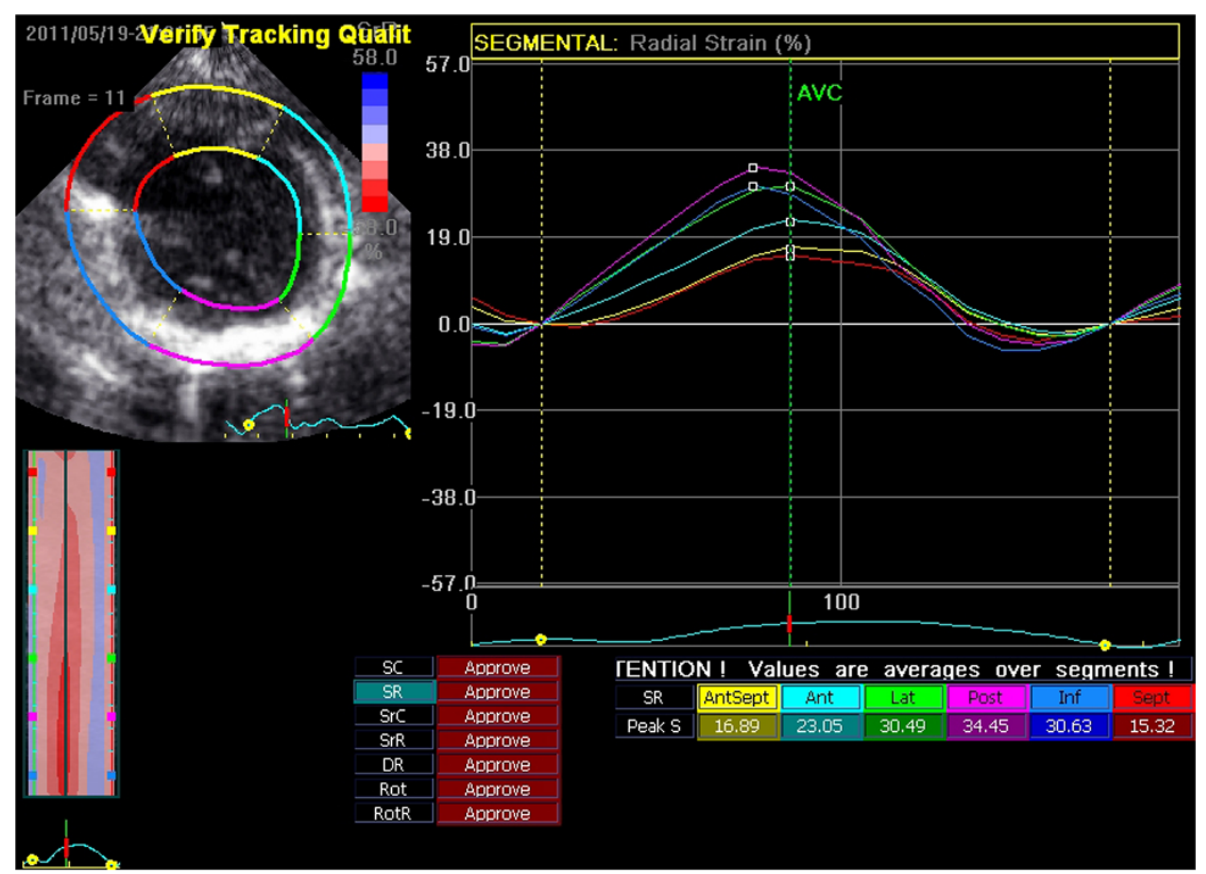

Figure 1 Systolic radial stain of the six myocardial segments.

published data has described that STE could detect subtle changes in LV function at an early subclinical stage [10-13].

The aim of the present study was to elucidate whether dietary hypercholesterolemia alters LV systolic function independently of CAD using STE in rabbits model and investigate their relationship.

\section{Methods}

\section{Animal model}

The experimental protocol was approved by a local ethical committee (First Affiliated Hospital, Xinjiang Medical University, Xinjiang, China). Twenty-eight male New Zealand rabbits (1.9-2.3 kg) were housed in separate cages in an environmentally controlled facility (AAALAC accredited) and were given water ad libitum and received humane care in compliance with institutions guidelines. The rabbits were acclimatized to laboratory conditions for 7 days prior to treatment. Eight rabbits were fed normal chow for 3 months as control (group 1) and the remaining 20 accepted an atherogenic diet for 2 months (group 2) or
3 months (group 3). The atherogenic diet contained $84 \%$ standard chow diet, $5 \%$ lard, $5 \%$ egg yolk powder and $2 \%$ cholesterol [13]. Diarrhea, appetite and coat color were observed during the experimental period.

\section{Echocardiographic imaging}

On the day of the study, rabbits fast for approximately $4 \mathrm{~h}$ to reduce abdominal distention and to facilitate obtaining the images. Echocardiographic images were acquired after lightly sedated with $10 \mathrm{mg} / \mathrm{kg}$ ketamine (Fujian Gutian Pharmaceutical Co., Ltd, China), $1 \mathrm{mg} / \mathrm{kg}$ Diazepam (Tianjin Jinyao Amino Acid Co., Ltd, China) and $0.025 \mathrm{mg} / \mathrm{kg}$ Atropine (Tianjin Pharmaceutical Group Co., Ltd, China) administered intravenously. The rabbits were placed in prone position without restraint. All images were obtained using a commercial ultrasound machine (Vivid 7 Dimension; GE Vingmed Ultrasound AS, Horten, Norway) with an M5S probe. M- mode images of parasternal long-axis view, B-mode images of apical three-chamber, four-chamber, and two-chamber views, short-axis views at the level of the mitral valve,

Table 1 General characteristics of the study animals

\begin{tabular}{llllll}
\hline Parameters & Group $\mathbf{1} \mathbf{n}=\mathbf{8}$ & Group $\mathbf{2} \mathbf{n}=\mathbf{1 0}$ & Group $\mathbf{3} \mathbf{n}=\mathbf{9}$ & F-value & $\boldsymbol{P}$-value \\
\hline HR (beats/min) & $183.50 \pm 9.49$ & $168.30 \pm 9.32$ & $187.89 \pm 11.02$ & 2.21 & 0.13 \\
SBP $(\mathrm{mmHg})$ & $109.88 \pm 11.31$ & $110.50 \pm 8.37$ & $111.22 \pm 10.33$ & 0.12 & 0.88 \\
DBP $(\mathrm{mmHg})$ & $78.25 \pm 8.05$ & $77.50 \pm 10.84$ & $82.67 \pm 10.06$ & 0.73 & 0.49 \\
\hline
\end{tabular}

$\mathrm{HR}$, heart rate; SBP, systolic blood pressure; $\mathrm{DBP}$, diastolic blood pressure. 
Table 2 Comparison of echocardiographic parameters

\begin{tabular}{llllll}
\hline Parameters & $\begin{array}{l}\text { Group1 } \\
\mathbf{n = 8}\end{array}$ & $\begin{array}{l}\text { Group2 } \\
\mathbf{n = 1 0}\end{array}$ & $\begin{array}{l}\text { Group3 } \\
\mathbf{n = 9}\end{array}$ & F-value & $\boldsymbol{P}$-value \\
\hline LVEDd $(\mathrm{mm})$ & $13.14 \pm 1.43$ & $13.51 \pm 1.69$ & $13.33 \pm 1.58$ & 0.12 & 0.89 \\
LVEDs $(\mathrm{mm})$ & $8.62 \pm 0.86$ & $9.00 \pm 1.02$ & $8.81 \pm 0.92$ & 0.37 & 0.70 \\
IVS $(\mathrm{mm})$ & $2.12 \pm 0.31$ & $1.88 \pm 0.36$ & $2.08 \pm 0.37$ & 1.28 & 0.30 \\
PW (mm) & $2.04 \pm 0.22$ & $2.01 \pm 0.20$ & $2.23 \pm 0.38$ & 1.63 & 0.22 \\
LVEF (\%) & $67.38 \pm 4.63$ & $65.97 \pm 4.61$ & $66.93 \pm 3.73$ & 0.25 & 0.78 \\
LAD (mm) & $8.84 \pm 1.15$ & $9.94 \pm 1.23$ & $9.42 \pm 0.99$ & 0.66 & 0.53 \\
\hline
\end{tabular}

LVEDd, left-ventricular end-diastolic diameter; LVEDs, left-ventricular endsystolic diameter; EF, ejection fraction; LAD, left-atrial diameter; IVS, ventricular septal end-diastolic thickness; PW, posterior wall end-diastolic thickness.

papillary muscles, and apex were obtained and digitally stored in cine-loop format for off line analysis [14]. LV end-diastolic diameter (LVEDd), LV end-systolic diameter (LVEDs), septal and LV posterior wall thickness, and left atrial anteroposterior diameter (LAD) were measured from standard planes. LV ejection fraction (EF) was calculated with the Teicholz formula [15].

\section{Strain analysis}

Two-dimensional B-mode images were captured with a frame rate of 50-80 fps and five beats were recorded for analysis. Blinded offline analyses of the short-axis views and apical long-axis views were performed using EchoPAC PC version 6.1.1 (GE Vingmed Ultrasound AS, Horten, Norway). After selecting the bestquality image of the cardiac cycle, the LV endocardial border was manually traced at the end-systolic frame, from which a speckle-tracking region of interest was automatically selected to approximate the myocardium between the endocardium and epicardium [16]. The workstation then computed and generated strain curves. The software automatically divided the sectional image into six segments according to the statement of the Cardiac Imaging Committee of the Council on Clinical Cardiology of the American Heart Association [17] (Figure 1). Strain curves of three consecutive cardiac cycles and values were imported for further analysis. To determine global longitudinal, circumferential and radial strain, the strain values of the 18 segments

Table 3 Peak systolic longitudinal strain values

\begin{tabular}{|c|c|c|c|c|c|}
\hline Longitudinal strain (\%) & Group $1 \mathrm{n}=8$ & Group $2 \mathrm{n}=10$ & Group $3 \mathrm{n}=9$ & F-value & $P$-value \\
\hline \multicolumn{6}{|l|}{ A4C septum } \\
\hline Basal segment & $-21.76 \pm 3.28$ & $-21.97 \pm 4.08$ & $-18.87 \pm 2.15$ & 2.240 & 0.129 \\
\hline Mid segment & $-23.40 \pm 4.38$ & $-21.89 \pm 3.47$ & $-18.91 \pm 2.20^{*}$ & 3.493 & 0.047 \\
\hline Apical segment & $-24.54 \pm 5.57$ & $-22.08 \pm 4.32$ & $-19.41 \pm 3.06^{*}$ & 2.685 & 0.090 \\
\hline \multicolumn{6}{|l|}{ A4C lateral wall } \\
\hline Basal segment & $-23.64 \pm 4.44$ & $-21.96 \pm 3.31$ & $-18.30 \pm 1.69^{* \boldsymbol{\Lambda}}$ & 5.382 & 0.019 \\
\hline Mid segment & $-20.88 \pm 3.74$ & $-20.98 \pm 3.20$ & $-18.32 \pm 2.01$ & 1.945 & 0.166 \\
\hline Apical segment & $-22.94 \pm 3.36$ & $-21.90 \pm 3.48$ & $-19.28 \pm 4.21$ & 2.115 & 0.143 \\
\hline \multicolumn{6}{|l|}{$\mathrm{A} 3 \mathrm{C}$ posterior wall } \\
\hline Basal segment & $-22.74 \pm 3.21$ & $-20.89 \pm 2.38$ & $-18.87 \pm 3.35^{*}$ & 3.425 & 0.050 \\
\hline Mid segment & $-21.71 \pm 2.68$ & $-22.05 \pm 2.65$ & $-18.57 \pm 3.13^{* \boldsymbol{\Lambda}}$ & 3.924 & 0.034 \\
\hline Apical segment & $-23.77 \pm 2.78$ & $-21.15 \pm 3.47$ & $-21.60 \pm 4.27$ & 1.327 & 0.285 \\
\hline \multicolumn{6}{|l|}{$\mathrm{A} 3 \mathrm{C}$ anterior septum } \\
\hline Basal segment & $-21.31 \pm 3.34$ & $-20.92 \pm 3.54$ & $-19.41 \pm 2.79$ & 0.764 & 0.477 \\
\hline Mid segment & $-21.96 \pm 2.95$ & $-21.09 \pm 3.70$ & $-19.62 \pm 3.68$ & 0.837 & 0.446 \\
\hline Apical segment & $-22.28 \pm 2.24$ & $-22.59 \pm 2.87$ & $-18.86 \pm 3.55^{* \boldsymbol{\Lambda}}$ & 4.197 & 0.028 \\
\hline \multicolumn{6}{|l|}{$\mathrm{A} 2 \mathrm{C}$ inferior wall } \\
\hline Basal segment & $-25.78 \pm 4.34$ & $-20.90 \pm 2.74^{*}$ & $-19.36 \pm 3.19^{*}$ & 7.749 & 0.003 \\
\hline Mid segment & $-23.37 \pm 4.53$ & $-20.02 \pm 2.59$ & $-19.08 \pm 3.61^{*}$ & 3.205 & 0.059 \\
\hline Apical segment & $-24.54 \pm 4.51$ & $-21.23 \pm 2.34$ & $-21.57 \pm 4.23$ & 2.027 & 0.155 \\
\hline \multicolumn{6}{|l|}{$\mathrm{A} 2 \mathrm{C}$ anterior wall } \\
\hline Basal segment & $-23.64 \pm 4.28$ & $-21.75 \pm 2.34$ & $-18.96 \pm 3.24^{*}$ & 4.066 & 0.031 \\
\hline Mid segment & $-23.17 \pm 3.39$ & $-23.37 \pm 2.68$ & $-17.82 \pm 3.00^{* \boldsymbol{\Lambda}}$ & 9.140 & 0.001 \\
\hline Apical segment & $-24.28 \pm 5.70$ & $-23.59 \pm 3.90$ & $-21.10 \pm 4.14$ & 1.075 & 0.358 \\
\hline
\end{tabular}

Data are expressed as mean \pm SD. A4C, Apical four-chamber; A3C, apical three-chamber; A2C, apical two-chamber. ${ }^{*} p<0.05$ for group 3 and group 2 vs. group 1, $\boldsymbol{\Delta}_{p}<0.05$ for group 3 vs. group 2. 
Table 4 Peak systolic circumferental strain values

\begin{tabular}{|c|c|c|c|c|c|}
\hline Circumferental strain (\%) & Group $1 \mathrm{n}=8$ & Group $2 n=10$ & Group $3 n=9$ & $F$-value & $P$-value \\
\hline \multicolumn{6}{|l|}{ Mitral valve level } \\
\hline Anteroseptal wall & $-30.18 \pm 5.52$ & $-30.33 \pm 5.91$ & $-23.45 \pm 6.55^{* \boldsymbol{\Lambda}}$ & 3.571 & 0.045 \\
\hline Anterior wall & $-23.76 \pm 4.86$ & $-23.22 \pm 4.42$ & $-20.09 \pm 2.33$ & 1.939 & 0.167 \\
\hline Lateral wall & $-20.63 \pm 5.89$ & $-19.75 \pm 3.35$ & $-19.10 \pm 3.19$ & 0.263 & 0.771 \\
\hline Posterior wall & $-21.40 \pm 4.46$ & $-20.85 \pm 4.58$ & $-18.65 \pm 3.25$ & 0.869 & 0.433 \\
\hline Inferior wall & $-22.58 \pm 4.22$ & $-20.89 \pm 4.59$ & $-17.34 \pm 3.73^{*}$ & 3.215 & 0.059 \\
\hline Septal wall & $-27.99 \pm 6.30$ & $-27.83 \pm 6.71$ & $-21.75 \pm 4.45^{* \boldsymbol{\Lambda}}$ & 2.937 & 0.073 \\
\hline \multicolumn{6}{|l|}{ Papillary level } \\
\hline Anteroseptal wall & $-28.22 \pm 7.03$ & $-29.73 \pm 6.00$ & $-24.80 \pm 4.06$ & 1.619 & 0.22 \\
\hline Anterior wall & $-20.58 \pm 3.78$ & $-22.99 \pm 4.44$ & $-19.76 \pm 2.89$ & 1.776 & 0.192 \\
\hline Lateral wall & $-18.99 \pm 3.19$ & $-20.18 \pm 3.17$ & $-18.31 \pm 2.04$ & 0.982 & 0.39 \\
\hline Posterior wall & $-19.32 \pm 3.52$ & $-19.73 \pm 3.31$ & $-19.45 \pm 1.06$ & 0.047 & 0.954 \\
\hline Inferior wall & $-21.01 \pm 3.60$ & $-20.59 \pm 2.33$ & $-17.19 \pm 1.89^{* \boldsymbol{\Lambda}}$ & 5.047 & 0.015 \\
\hline Septal wall & $-27.98 \pm 7.59$ & $-28.21 \pm 5.53$ & $-20.66 \pm 3.03^{* \boldsymbol{\Lambda}}$ & 4.717 & 0.019 \\
\hline \multicolumn{6}{|l|}{ Apical level } \\
\hline Anteroseptal wall & $-30.24 \pm 6.67$ & $-28.71 \pm 6.29$ & $-21.53 \pm 3.78^{* \boldsymbol{\Lambda}}$ & 5.284 & 0.013 \\
\hline Anterior wall & $-25.66 \pm 5.46$ & $-23.68 \pm 5.03$ & $-19.68 \pm 2.60^{*}$ & 3.539 & 0.046 \\
\hline Lateral wall & $-24.88 \pm 4.33$ & $-22.72 \pm 6.18$ & $-19.48 \pm 3.25^{*}$ & 2.192 & 0.134 \\
\hline Posterior wall & $-25.33 \pm 5.92$ & $-23.65 \pm 3.84$ & $-19.49 \pm 3.06^{*}$ & 3.782 & 0.038 \\
\hline Inferior wall & $-25.81 \pm 5.45$ & $-23.46 \pm 5.93$ & $-18.66 \pm 2.86^{*}$ & 4.228 & 0.027 \\
\hline Septal wall & $-26.64 \pm 6.78$ & $-27.42 \pm 6.34$ & $-24.85 \pm 5.08$ & 0.553 & 0.582 \\
\hline
\end{tabular}

Data are expressed as mean \pm SD. ${ }^{*} \mathrm{p}<0.05$ for group 3 and group 2 vs. group $1,{ }^{\mathbf{\Delta}} \mathrm{p}<0.05$ for group 3 vs. group 2 .

were averaged for the apical views or the short-axis views.

\section{Blood pressure measurement}

After echocardiographic imaging, blood pressure measurements were made from the central ear artery (CEA) of rabbits using a 20G vascular catheter (Johnson and Johnson, Belgium). The arterial catheter was connected to a pressure transducer (MLT0699, AD Instruments, Pty Ltd, Australia) and an analog-to-digital converter (PowerLab, ML866, AD Instruments, Pty Ltd, Australia). Arterial systolic and diastolic pressure were recorded.

\section{Cholesterol analysis}

Peripheral blood was collected from ear veins with a 25 -gauge needle and syringe at baseline, 2 months and 3 months. Total cholesterol (TC) and low density lipoprotein cholesterol (LDL-C) were measured with an automated clinical chemistry analyzer (AU680, Beckman Instruments, USA). All rabbits were euthanized at correspnding time and the hearts removed. A segment of myocardial tissue $(10 \times 3 \mathrm{~mm})$ from the LV free wall was excised. Cholesterol levels were measured in the tissue segment using a cholesterol Kit (EnzyChrom Cholesterol Assay Kit, BioAssay Systems, Hayward, CA).

\section{Statistical analysis}

SPSS 16.0 (SPSS inc., Chicago, Illinois, USA) was used for statistical analysis. The data were tested for normality and homogeneity of variance. Data are expressed as mean \pm standard deviation (SD). One way ANOVA was used to compare the echocardiographic parameters, strain parameters, myocardial and serum cholesterol levels for all three groups. Pearson correlation analysis was done between STE variables and cholesterol levels. For all analyses, a $P$ value $<0.05$ was considered significant. Interobserver and intraobserver variability for strain measurements were examined using both Pearson's bivariate two-tailed correlations and Bland-Altman analysis from 10 randomly selected rabbits.

\section{Results}

Animal

Of the 28 experimental rabbits, 1 died in the group 3 due to diarrhea. Heart rates and blood pressure of three groups were similar $(P>0.05$, Table 1$)$. 
Table 5 Peak systolic radial strain values

\begin{tabular}{|c|c|c|c|c|c|}
\hline Radial strain (\%) & Group $1 \mathrm{n}=8$ & Group $2 \mathrm{n}=10$ & Group $3 n=9$ & $F$-value & $P$-value \\
\hline \multicolumn{6}{|l|}{ Mitral valve level } \\
\hline Anteroseptal wall & $36.84 \pm 6.98$ & $38.88 \pm 6.16$ & $45.48 \pm 6.85^{* \boldsymbol{\Lambda}}$ & 3.756 & 0.039 \\
\hline Anterior wall & $37.92 \pm 7.15$ & $40.48 \pm 8.06$ & $44.77 \pm 9.82$ & 1.368 & 0.275 \\
\hline Lateral wall & $41.65 \pm 7.78$ & $42.02 \pm 9.12$ & $46.48 \pm 9.37$ & 0.765 & 0.477 \\
\hline Posterior wall & $39.65 \pm 5.41$ & $44.31 \pm 7.06$ & $49.07 \pm 6.21^{*}$ & 4.426 & 0.024 \\
\hline Inferior wall & $40.92 \pm 5.31$ & $42.54 \pm 7.64$ & $50.13 \pm 7.02^{* \boldsymbol{\Lambda}}$ & 4.231 & 0.027 \\
\hline Septal wall & $38.09 \pm 7.21$ & $40.84 \pm 7.21$ & $47.40 \pm 5.41^{*}$ & 4.098 & 0.03 \\
\hline \multicolumn{6}{|l|}{ Papillary level } \\
\hline Anteroseptal wall & $37.41 \pm 5.01$ & $38.11 \pm 5.38$ & $44.81 \pm 10.12^{*}$ & 2.726 & 0.087 \\
\hline Anterior wall & $37.06 \pm 7.45$ & $37.83 \pm 6.56$ & $39.28 \pm 7.31$ & 0.205 & 0.816 \\
\hline Lateral wall & $42.32 \pm 7.81$ & $39.45 \pm 7.27$ & $42.05 \pm 9.37$ & 0.351 & 0.708 \\
\hline Posterior wall & $42.54 \pm 7.14$ & $44.24 \pm 3.81$ & $46.22 \pm 11.70$ & 0.432 & 0.654 \\
\hline Inferior wall & $43.18 \pm 8.54$ & $42.40 \pm 4.87$ & $45.67 \pm 6.38$ & 0.574 & 0.571 \\
\hline Septal wall & $39.85 \pm 8.45$ & $41.14 \pm 6.87$ & $48.49 \pm 6.96^{* \boldsymbol{\Lambda}}$ & 3.23 & 0.058 \\
\hline \multicolumn{6}{|l|}{ Apical level } \\
\hline Anteroseptal wall & $39.10 \pm 6.70$ & $38.63 \pm 5.90$ & $43.96 \pm 6.63$ & 1.802 & 0.187 \\
\hline Anterior wall & $37.29 \pm 6.44$ & $38.84 \pm 5.84$ & $44.71 \pm 4.59^{* \boldsymbol{\Lambda}}$ & 3.88 & 0.035 \\
\hline Lateral wall & $43.85 \pm 8.84$ & $40.85 \pm 3.97$ & $47.66 \pm 9.67^{* \boldsymbol{\Lambda}}$ & 3.159 & 0.061 \\
\hline Posterior wall & $41.17 \pm 7.37$ & $41.74 \pm 6.88$ & $44.07 \pm 12.15$ & 0.241 & 0.788 \\
\hline Inferior wall & $44.52 \pm 6.66$ & $42.14 \pm 8.85$ & $44.90 \pm 7.49$ & 0.336 & 0.718 \\
\hline Septal wall & $42.44 \pm 6.06$ & $38.34 \pm 5.84$ & $46.96 \pm 10.41^{\wedge}$ & 2.88 & 0.076 \\
\hline
\end{tabular}

Data are expressed as mean \pm SD. ${ }^{*} p<0.05$ for group 3 and group 2 vs. group $1,{ }^{\mathbf{4}} p<0.05$ for group 3 vs. group 2 .
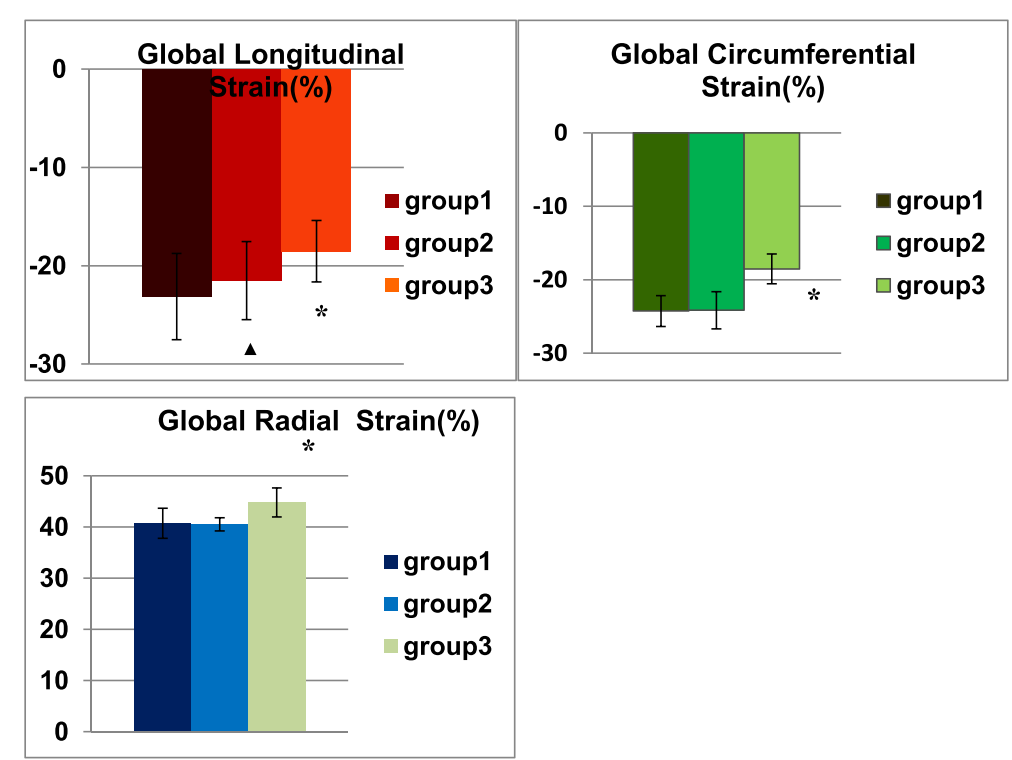

Figure 2 Global peak systolic longitudinal, circumferential and radial strain in the three groups. ${ }^{*} p<0.001$ for group 3 vs. group 2 and group 1. $\mathrm{A}<0.05$ for group 2 vs group 1 . 
Table 6 Serum and tissue cholesterol profiles

\begin{tabular}{lccccc}
\hline Parameters & Group $\mathbf{1} \mathbf{n}=\mathbf{8}$ & Group $\mathbf{2} \mathbf{n}=\mathbf{1 0}$ & Group $\mathbf{3} \mathbf{~ n = 9}$ & F-value & $\boldsymbol{P}$-value \\
\hline Serum TC $(\mathrm{mmol} / \mathrm{L})$ & $2.07 \pm 0.60$ & $24.15 \pm 5.36^{*}$ & $34.74 \pm 10.40^{* \mathbf{\Lambda}}$ & 191.27 & 0.000 \\
Serum LDL-C $(\mathrm{mmol} / \mathrm{L})$ & $1.13 \pm 0.54$ & $10.73 \pm 3.32^{*}$ & $31.62 \pm 3.68^{* \mathbf{\Delta}}$ & 229.60 & 0.000 \\
Tissue cholesterol $(\mu \mathrm{mol} / \mathrm{g})$ & $0.86 \pm 0.29$ & $2.22 \pm 0.62^{*}$ & $4.92 \pm 1.63^{* \mathbf{\Delta}}$ & 34.68 & 0.000 \\
\hline
\end{tabular}

Data are expressed as mean \pm SD. TC, total cholesterol; LDL-C, low density lipoprotein cholesterol.

${ }^{*} \mathrm{p}<0.05$ for group 3 and group 2 vs. group $1,{ }^{\star} \mathrm{p}<0.05$ for group 3 vs. group 2 .

\section{Conventional echocardiography}

Echocardiographic measurements of the different groups were shown in Table 2. There were no significant differences in LVEF, LVEDd, LVEDs, IVS, PW and LAD among three groups $(P>0.05)$.

\section{Strain measurements}

From a total of 972 analyzed segments, 28 segments were excluded owing to suboptimal myocardial tracking and poor image quality. Regional longitudinal (Table 3) and circumferential (Table 4) strain of LV were significantly reduced in group 3 compared with group 1 and 2. But regional radial strain of LV were significantly increased in group 3 (Table 5).

Global longitudinal myocardial deformation of the LV was significantly impaired both in group 2 and 3, while radial deformation was increased in group 3 compared with group 1 and 2 . In addition, global circumferential strain was also reduced in group 3 compared with group 1 and 2 (Figure 2).

\section{Serum and tissue cholesterol profiles}

The serum cholesterol profiles of the three groups after experiment were shown in Table 6. There was a statistically significant increase in serum TC, LDL-C and tissue cholesterol levels in animals fed with cholesterol enriched diet compared with the control group $(\mathrm{p}<0.05)$. Morerove, the concentration of cholesterol increased with feeding duration $(p<0.05)$.

\section{Correlation between strain parameters and cholesterol levels}

The correlation between strain parameters and cholesterol were shown in Table 7. There was significant inverse

Table 7 Correlation between strain paramters and cholesterol

\begin{tabular}{|c|c|c|c|c|c|c|}
\hline \multirow{2}{*}{$\begin{array}{l}\text { Strain } \\
\text { parameters }\end{array}$} & \multicolumn{2}{|c|}{ Serum TC } & \multicolumn{2}{|c|}{ Serum LDL -C } & \multicolumn{2}{|c|}{ Tissue cholesterol } \\
\hline & $r$ & $p$ & $r$ & $p$ & $r$ & $p$ \\
\hline GLS & -0.723 & 0.000 & -0.794 & 0.000 & -0.700 & 0.000 \\
\hline GRS & 0.432 & 0.028 & 0.602 & 0.001 & 0.469 & 0.016 \\
\hline GCS & -0.518 & 0.007 & -0.691 & 0.000 & -0.659 & 0.000 \\
\hline
\end{tabular}

GLS, global longitudinal strain; GRS, global radial strain; GCS, global circumferential strain; TC, total cholesterol; LDL-C, low density lipoprotein cholesterol. correlation between global longitudinal strain and serum TC, LDL-C as well as myocardial cholesterol levels. $(r=-0.723, \mathrm{P}<0.001 ; \mathrm{r}=-0.794, \mathrm{P}<0.001 ; \mathrm{r}=-0.70$, $\mathrm{P}<0.001$ ). A significant negative correlation was also noted between global circumferential strain and serum TC, LDL-C as well as myocardial cholesterol levels. $(\mathrm{r}=-0.518, \mathrm{P}=0$.007; $\mathrm{r}=-0.691, \mathrm{P}<0.001$; $\mathrm{r}=-0.659, \mathrm{P}<0.001)$. A significant positive correlation was found between radial strain and serum TC, LDL-C as well as myocardial cholesterol levels. $(r=0.432, P=0.028$; $\mathrm{r}=0.602, \mathrm{P}=0.001 ; \mathrm{r}=0.469, \mathrm{P}=0.016$ ).

\section{Reproducibility}

The results showed very good intra-observer variability for longitudinal, circumferential and radial strain rate $(\mathrm{r}=$ 0.817, $\mathrm{P}=0.004 ; \mathrm{r}=0.798, \mathrm{P}=0.006 ; \mathrm{r}=0.868, \mathrm{P}=0.001$ ). The Bland-Altman plots demonstrated acceptable interobserver variability for all strain parameters (Figure 3).

\section{Discussion}

To the best of our knowledge, the present study is the first to comprehensively compare strain parameters-derived STI with the levels of serum and myocardial cholesterol in diet-induced expeimental hypercholesterolemia. Although previous experimental studies have shown that dietinduced hypercholesterolemia resulted in contractile reduction of single ventricular myocyte without any change in pressure or LV morphology [4], few data are available from in vivo investigations. The present study displayed the application of STE as a noninvasive imaging technique to elucidate the direct effect of hypercholesterolemia on LV myocardial deformation in a rabbit model.

In our study, there were no significant differences in LV morphology, EF and blood pressure among groups, whereas LV strain was found to be reduced in the hypercholesterolemic rabbits. Previous studies failed to show abnormalities using EF, which may be due to EF reflects the whole LV systolic function, under the influence of pre and afterload [18]. With the application of advanced techniques, such as strain, strain rate, incipient systolic dysfunction has been detected in subclinical diseases [19]. Moreover, our analysis indicates that longitudinal dysfunction are the first appeared, followed by circumferential, which suggest the importance of longitudinal strain in the assessment of LV systolic dysfunction in subclinical stage. 


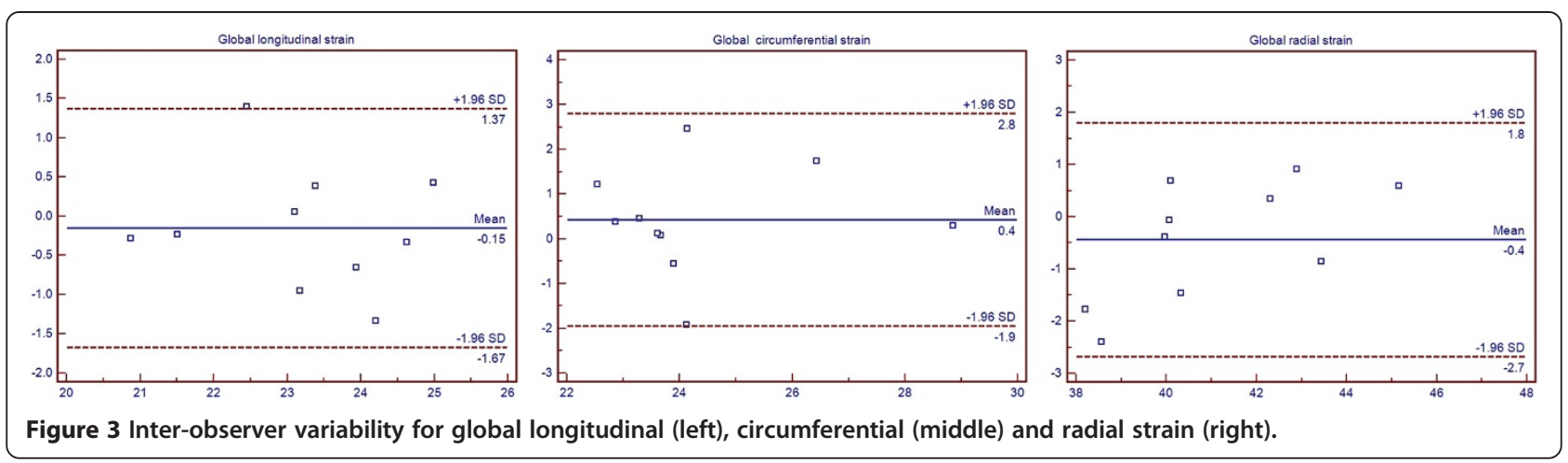

Whether similar changes occur in humans with hypercholesterolemia can not confirm from our study. However, a recent human study demontrated longitudinal and circumferential deformations were both impaired in the children with heterozygous familiar hypercholesterolemia [20]. Thus, we believe that the abnormalities we found in rabbit models with hypercholesterolemia indicate an early sign of hypercholesterolemiainduced myocardial dysfunction, in agreement with the in vitro expriments [4].

Interestingly, our study demonstrates that the increased radial deformation make up for impaired longitudinal and circumferential strain in rabbit hypercholesterolemic models to maintain LVEF. This finding is consistent with prior reports in children with heterozygous familial hypercholesterolemia and other preclinical diseases $[14,20,21]$. The potential mechanism by which hypercholesterolemia causes the increase in radial deformation remains unclear. A possible explanation could be the realignments of myocardial fiber orientation in the outer half of the myocardium may contribute to "transmural compensation" by less impaired epicardial fibers [22].

In the present study, a significant negative correlation were found between global longitudinal strain and serum cholesterol level as well as myocardial cholesterol levels. These results indicate that the cholesterol accumulated in the myocardium may be responsible for a reduction in myocardial strain. Similar to our study, Wang et al. [23] reported a positive correlation between serum HDL levels and LVEF in human subjects with serum hypercholesterolemia even in the absence of angiographic evidence of CAD.

The precise mechanism responsible for the association between cholesterol level and impaired myocardial deformation cannot be determined from our study. However, several mechanisms have been proposed to explain LV dysfunction induced by hypercholesterolemia: (1) increased cardiac oxidative stress [24], (2) alteration of the myocardial energy metabolism [22], (3) changes in myosin heavy-chain isoform expression patterns [4], (4) down-regulation and redistribution of connexin-43 expression in myocardium [25], and (5) impaired activation of myocardial adenosine triphosphate-sensitive potassium channels [19]. These mechanisms may represent the basis for a "hypercholesterolemic cardiomyopathy [26].

\section{Study limitations}

As a limitations of our study, administration of ketamineDiazepam - Atropine combinations induces mild bradycardia, which slightly alters cardiac function. In addition, LV diastolic function, rotation and torsion mechanics are potentially very important features for the comprehensive understanding of myocardial tissue damage; therefore, lack of measurement of diastolic function, rotation and torsion was another limitation of the present study.

\section{Conclusion}

Hypercholesterolemia was significantly associated with LV myocardial functional alterations apart from CAD. The findings also indicate that decreases in longitudinal was the first appeared, followed by circumferential, and was compensated for by increasing radial strain. Thus, the application of STE may provide noninvasive functional insight into disease progression or recovery in reponse to therapeutic intervention.

\section{Abbreviations}

CAD: Coronary artery disease; STE: Speckle-tracking echocardiography; LV: Left ventricular; TC: Total cholesterol; LDL-C: Low density lipoprotein cholesterol; LVEDd: LV end-diastolic diameter; LVEDs: LV end-systolic diameter; CEA: Central ear artery.

\section{Competing interests}

The authors declare that they have no competing interests.

\section{Authors' contribution}

The authors contributions were as follows: YM designed the study and revised the first manuscript draft. LL obtained all echocardiographic images, performed statistical analysis and wrote the first manuscript draft. CW and JW performed the echocardiographic analysis. All authors have read and approved the final manuscript. 


\section{Acknowledgement}

This study was supported by the National Natural Science Foundation of China (No. 81060121). The authors wish to thank Tao Jiang for excellent technical assistance.

Received: 4 March 2014 Accepted: 1 August 2014

Published: 9 August 2014

\section{References}

1. He J, Ogden LG, Bazzano LA, Vupputuri S, Loria C, Whelton PK: Risk factors for congestive heart failure in US men and women: NHANES I epidemiologic follow-up study. Arch Intern Med 2001, 161:996-1002.

2. Conroy RM, Pyörälä K, Fitzgerald AP, Sans S, Menotti A, De Backer G, De Bacquer D, Ducimetière P, Jousilahti P, Keil U, Njølstad I, Oganov RG, Thomsen $T$, Tunstall-Pedoe $H$, Tverdal A, Wedel H, Whincup P, Wilhelmsen L, Graham IM: The SCORE project group: estimation of ten-year risk of fatal cardiovascular disease in Europe: the SCORE project. Eur Heart I 2003, 24:987-1003.

3. Kitamura A, Noda H, Nakamura M, Kiyama M, Okada T, Imano H, Ohira T, Sato S, Yamagishi K, Iso H: Association between non-high-density lipoprotein cholesterol levels and the incidence of coronary heart disease among Japanese: the Circulatory Risk in Communities Study (CIRCS). J Atheroscler Thromb 2011, 18:454-463.

4. Huang Y, Walker KE, Hanley F, Narula J, Houser SR, Tulenko TN: Cardiac systolic and diastolic dysfunction after a cholesterol-rich diet. Circulation 2004, 109:97-102.

5. Luo TY, Su MJ, Yang YF, Liu YB, Liang HC, Wu CC, Lee YT: Effect of hypercholesterolemia on myocardial function in New Zealand white rabbits. J Biomed Sci 2004, 11:829-837.

6. Baigent C, Keech A, Kearney PM, Blackwell L, Buck G, Pollicino C, Kirby A, Sourjina T, Peto R, Collins R, Simes R, Cholesterol Treatment Trialists' (CTI) Collaborators: Efficacy and safety of cholesterollowering treatment: prospective meta-analysis of data from 90056 participants in 14 randomised trials of statins. Lancet 2005, 366:1267-1278.

7. Blessberger $\mathrm{H}$, Binder $\mathrm{T}$ : Non-invasive imaging: two dimensional speckle tracking echocardiography-basic principles. Heart 2010, 96:716-722.

8. Geyer H, Caracciolo G, Abe H, Wilansky S, Careri S, Gentile F, Nesser HJ, Khandheria B, Narula J: Assessment of myocardial mechanics using speckle tracking echocardiography: fundamentals and clinical applications. J Am Soc Echocardiogr 2010, 23:351-369.

9. Mondillo S, Galderisi M, Mele D, Cameli M, Lomorilello VS, Zacà V, Ballo P, D'Andrea A, Muraru D, Losi M, Agricola E, D'Errico A, Buralli S, Sciomer S, Nistri S, Badano L: Speckle-tracking echocardiography: a new technique for assessing myocardial function. J Ultrasound Med 2011, 30:71-83.

10. Lipiec P, Szymczyk E, Michalski B, Stefańczyk L, Woźniakowski B, Rotkiewicz A, Szymczyk K, Kasprzak JD: Echocardiographic quantitative analysis of resting myocardial function for the assessment of viability after myocardial infarction-comparison with magnetic resonance imaging. Kardiol Pol 2011, 69:915-922.

11. Kusunose K, Yamada H, Nishio S, Mizuguchi Y, Choraku M, Maeda Y, Hosokawa S, Yamazaki N, Tomita N, Niki T, Koshiba K, Soeki T, Wakatsuki T, Akaike M, Sata M: Validation of longitudinal peak systolic strain by speckle tracking echocardiography with visual assessment and myocardial perfusion SPECT in patients with regional asynergy. Circ $J$ 2011, 75:141-147.

12. Lancellotti P, Cosyns B, Zacharakis D, Attena E, Van Camp G, Gach O, Radermecker M, Piérard LA: Importance of left ventricular longitudinal function and functional reserve in patients with degenerative mitral regurgitation: assessment by two-dimensional speckle tracking. J Am SOC Echocardiogr 2008, 21:1331-1336.

13. Li M, Zhang Y, Ren H: Effect of clopidogrel on the inflammatory progression of early atherosclerosis in rabbits model. Atherosclerosis 2009 194(2):348-356.

14. Di Salvo G, D'Aiello AF, Castaldi B, Fadel B, Limongelli G, D'Andrea A Pergola V, Pacileo G, Del Giudice EM, Perrone L, Calabrò R, Russo MG: Early left ventricular abnormalities in children with heterozygous familial hypercholesterolemia. J Am Soc Echocardiogr 2012, 25(10):1075-1082.

15. Vuille C, Weyman AE: Left Ventricle I: General Considerations, Assessment of Chamber Size and Function. In Principles and Practice of Echocardiography. 2nd edition. Edited by Vuille C, Weyman AE. Philadelphia (PA): Lea \& Febiger; 1994:575-611.
16. Marcus KA, Mavinkurve-Groothuis AMC, Barends M, van Dijk A, Feuth T, de Korte C, Kapusta L: Reference values for myocardial two-dimensional strain echocardiography in a healthy pediatric and young adult cohort. J Am Soc Echocardiogr 2011, 24:625-636.

17. Cerqueira MD, Weissman NJ, Dilsizian V, Jacobs AK, Kaul S, Laskey WK, Pennell DJ, Rumberger JA, Ryan T, Verani MS: American Heart AssociationWriting Group on Myocardial Segmentation and Registration for Cardiac Imaging. Standardized myocardial segmentation and nomenclature for tomographic imaging of the heart. A statement for healthcare professionals from the Cardiac Imaging Committee of the Council on Clinical Cardiology of the American Heart Association. Int J Cardiovasc Imaging 2002, 18:539-542.

18. Movahed M, Saito Y: Lack of association between obesity and left ventricular systolic dysfunction. Echocardiography 2009, 26:128-132.

19. Lee TM, Chou TF, Tsai CH: Association of pravastatin and left ventricular mass in hypercholesterolemic patients: role of 8-iso-prostaglandin F2a formation. J Cardiovasc Pharmacol 2002, 40:868-874.

20. Nakai H, Takeuchi M, Nishikage T, Lang RM, Otsuji Y: Subclinical left ventricular dysfunction in asymptomatic diabetic patients assessed by two dimensional speckle tracking echocardiography: correlation with diabetic duration. Eur J Echocardiogr 2009, 10:926-932.

21. Fang $Z Y$, Leano $R$, Marwick $T H$ : Relationship between longitudinal and radial contractility in subclinical diabetic heart disease. Clin Sci 2004 106:53-60.

22. lida N, Seo Y, Ishizu T, Nakajima H, Atsumi A, Yamamoto M, Machino-Ohtsuka T, Kawamura R, Enomoto M, Kawakami Y, Aonuma K: Compensation of myocardial deformation to preserve left ventricular ejection performance in chronic aortic regurgitation. J Am Soc Echocardiogr 2012, 25:620-628.

23. Wang YT, Popovic' ZB, Efimov IR, Cheng Y: Longitudinal study of cardiac remodelling in rabbits following infarction. Can J Cardio/ 2012, 28:230-238

24. Prasad K, McNair ED, Qureshi AM, Casper-Bell G: Vitamin E slows the progression of hypercholesterolemia-induced oxidative stress in heart, liver and kidney. Mol Cell Biochem 2012, 368:181-187.

25. Lin LC, Wu CC, Yeh HI, Lu LS, Liu YB, Lin SF, Lee YT: Down regulated myocardial connexin 43 and suppressed contractility in rabbits subjected to a cholesterol enriched diet. Lab Invest 2005, 85:1224-1237.

26. Talini E, Di Bello V, Bianchi C, Palagi C, Delle DMG, Penno G, Nardi C, Canale ML, Del Prato S, Mariani M, Miccoli R: Early impairment of left ventricular function in hypercholesterolemia and its reversibility after short term treatment with rosuvastatin: a preliminary echocardiographic study. Atherosclerosis 2008, $197 \cdot 346-354$

doi:10.1186/1476-511X-13-128

Cite this article as: Liu et al: Association of hypercholesterolemia and cardiac function evaluated by speckle tracking echocardiography in a rabbit model. Lipids in Health and Disease 2014 13:128.

\section{Submit your next manuscript to BioMed Central and take full advantage of:}

- Convenient online submission

- Thorough peer review

- No space constraints or color figure charges

- Immediate publication on acceptance

- Inclusion in PubMed, CAS, Scopus and Google Scholar

- Research which is freely available for redistribution 